\title{
The Properties of Tree-Like Hierarchical Networks
}

\author{
Yingxin Zhang* Chao Chen and Jianmai Shi \\ Science and Technology on Information Systems Engineering Laboratory, National University of Defense Technology, 410073,
} Changsha, Hunan, P. R. China

Received: 1 Mar. 2013, Revised: 15 Apr. 2013, Accepted: 19 Apr. 2013

Published online: 1 Nov. 2013

\begin{abstract}
Tree-like hierarchical structure is one of the key features in many real complex systems. To investigate its properties, a deterministic tree-like hierarchical network model is proposed. Next, the static properties of the tree-like hierarchical networks are discussed. The simulation and theoretical results prove that the artificial tree-like networks conformably possess small-world and scalefree properties well. Furthermore, the dynamic cascading failures in the tree-like hierarchical networks are investigated. A weighted tree-like cascading failure model is proposed, and the impacts of topological structure on the network robustness are investigated by a set of illustrative simulations.
\end{abstract}

Keywords: Tree-like hierarchical network, scale-free, small-world, cascading failures.

\section{Introduction}

Tree-like hierarchical structure is one of the key features in many real complex systems. It is well known in computer science of the algorithms and compilers [1,2], and has been one of the hot topics in network science [3, 4]. Most of these hierarchical models are constructed based on stochastic and computer simulations, i.e., new nodes connect using a certain rule to the nodes already present in the system. However, the randomness in stochastic models makes it confusing to gain a visual understanding of the topology of the network [5]. It would therefore be of interest to construct models that lead to tree-like networks in a deterministic fashion. Here we present such a simple model, generating a deterministic tree-like hierarchical network.

Small-word and scale-free properties are two common features in many real complex networks. We hence investigate the small-world and scale-free properties of the proposed tree-like hierarchical networks. The simulation and theoretical analysis prove that our proposed tree-like hierarchical networks conformably possess the small-world and scale-free properties well.

Network robustness against cascading failures is one of the most central topics in network safety [6]. Evidence has demonstrated that in many complex networks, even though initial failures emerge very locally, the entire network can be greatly affected, even resulting in the collapse of the whole system. Hence we investigate the robustness of the tree-like hierarchical networks against cascading failures. A weighted tree-like cascading failures model is first proposed, and then the impacts of the tree-like hierarchical structure function on the networks robustness are discussed.

The rest of this paper is organized as follows: in Section 2, we introduce the tree-like hierarchical network model and discuss its fundamental parameters. In Section 3 , we verify the small-world and scale-free properties of the artificial tree-like hierarchical networks. The tree-like cascading failures model and the networks' robustness against cascading failure are respectively discussed in Section 4 and 5. Finally, some concluding remarks of Section 6 end the paper.

\section{Tree-like hierarchical network model}

In our tree-like hierarchical network model, we emphasize two mechanisms: the phenomenon of "branch" and "level-skipping" conception, which are ubiquitous in tree-like hierarchical structures [7]. For example, in one regular military corps organization, the "branch" phenomenon could be stated as follows: one corps contains several divisions, one division contains several regiments, one regiment contains several battalions, and one battalion contains several companies or similar

\footnotetext{
*Corresponding author e-mail: zhyingxin@ 163.com
} 
subunits. In such a tree-like hierarchical organization, the regular leader ship levels from the top to the lowest ones may be: corps commander, division commanders, regiment commanders, battalion commanders, and company commanders. However, the corps commander could command his regiments directly by skipping the level of division; the division commanders could command their battalions directly by skipping the 'level' of regiment. In fact, such "level-skipping" phenomena are ubiquitous and easily accomplished, as they need to skip only one or few levels. On the other hand, it is relatively difficult to require the corps commander to directly command all his companies, as this need to skip four levels. In order to distinguish the capacity of "level-skipping", we introduce the conception of "Skipping-ability", which which will be defined in the following section.

In one university, the leader ship levels from the top to the lowest ones may be: president, department heads, staffs, and students [7]. Usually, the president may contact students through the levels of heads and staffs. However, he could also meet the students directly by skipping the levels when necessary. Similar behaviors may also exist in companies, factories, communication networks, power supply networks, actor collaboration networks, etc. Our tree-like hierarchical network model is primarily motivated by the above examples.

\subsection{Construction of the model}

In order to facilitate the description of tree-like hierarchical networks in this paper, we first introduce three definitions:

Branch $(B)$ : the maximum direct branches of each node in the tree-like hierarchical network.

Hierarchy $(H)$ : the total levels of the tree-like hierarchical network.

Skipping-ability $(S)$ : the maximum skipping levels of a tree-like hierarchical network.

A tree-like hierarchical network with branch $B$ equals $m$, hierarchy $H$ equals $n$ and skipping-ability $S$ equals $s$ is henceforth referred to simply as " $m-n-s$ " tree-like network.

Now we describe the construction procedure of the " $m$ $n-s$ " tree-like network. An illustrative example of "2-4-3" tree-like network is depicted in Figure 1. The procedure can be stated as follows.

Step 0: Initialize the network parameters $B=m, H=n$ and $S=s$. In Figure 1 , we initialize $B=2, H=4$ and $S=3$.

Step 1: We start from a single node, and let it to be the main root. The main root constitutes the first hierarchy. In Figure 1, the node 1 is generated being as the main root.

Step 2: Generate $m$ new nodes to constitute the second hierarchy of the network, and connect each of them to the main root. In Figure 1, node 2 and 3 are generated, and are connected to the main root node 1 respectively.

Step 3: Generate $m$ subordinate nodes for each of the second hierarchy node, and connect them to their

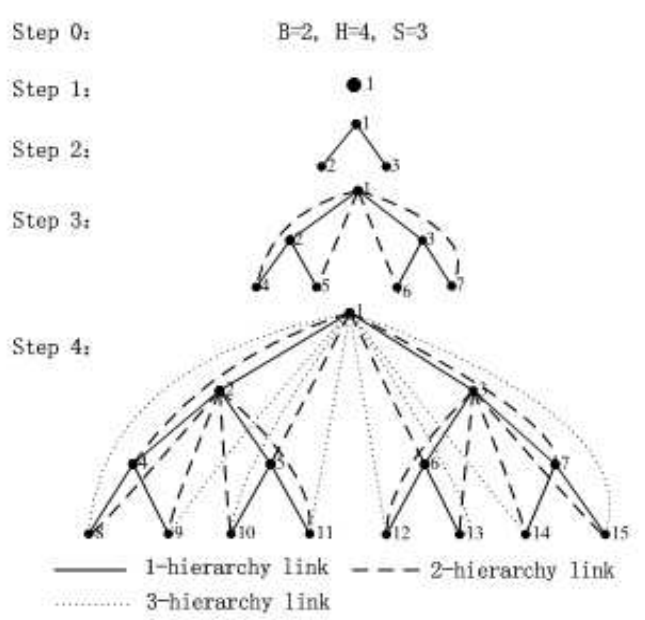

Fig. 1: Construction of the 2-4-3 tree-like network

maximum $s$ hierarchy ancestral nodes. In Figure 1, nodes 4 and 5 are generated for node 2 , and are connected to their common 1-hierarchy ancestral node 2 and 2-hierarchical ancestral node 1 respectively. Analogously, nodes 6 and 7 are generated for node 3 , and are connected to their common 1-hierarchy ancestral node 3 and 2-hierarchical ancestral node 1 respectively.

These rules can be easily generalized. In Step h, $m^{n-1}$ new nodes are added into the model, and each of them is connected to its maximum s hierarchy ancestral nodes. The operations are as follows:

Step h: Generate $m$ subordinate nodes for each of the above hierarchy node, and connect them to their maximum $c$ hierarchy ancestral nodes.

Finally, after s steps, an " $m-n-s$ " tree-like network is generated.

It should be aware that one kind of tree-like networks with the skipping-ability " $S=H$ " are ubiquitous in our society. To date, a large amount of attention has been paid to such networks. Chen et al. [7] propose a simple pseudo tree-like network model, deterministic complex network (DCN) model. In fact, the DCN model can be seen as a special case of our " $B-H-S$ " tree-like network with $\mathrm{B}=2$ and $\mathrm{S}=\mathrm{H}$. The authors have proved that the DCN model possesses the small-world and scale-free properties well. Therefore, it is of interest to verify the small-world and scale-free properties of our general " $m-n-n$ " tree-like networks. To facilitate the following description, the kind of " $m-n-n$ " tree-like network will be simply described as " $m-n$ " tree-like network.

\subsection{The fundamental parameters}

In this section, we discuss the fundamental parameters of the $m-n$ tree-like network. 


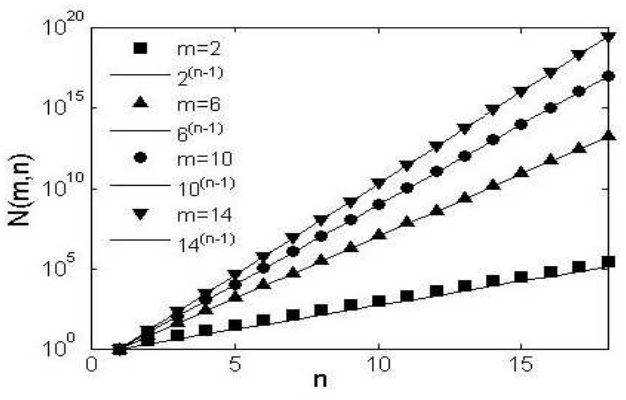

Fig. 2: Node sizes of the $m-n$ tree-like networks

$N(m, n)=1+m+m^{2}+\cdots+m^{n-1}=\frac{1-m^{n}}{1-m}$

$$
\begin{array}{r}
E(m, n)=1 \times\left(m+m^{2}+\cdots+m^{n-1}\right)+m \times \\
\left(m+m^{2}+\cdots+m^{n-2}\right)+m^{n-2} \times m \\
=m+2 m^{2}+\cdots+(n-1) m^{n-1}=\sum_{i=0}^{n-1} i \cdot m^{i}
\end{array}
$$

$$
\triangle N(m, n)=N(m, n)-N(m, n-1)=m^{n-1}
$$

$$
\triangle E(m, n)=E(m, n)-E(m, n-1)
$$

$=(n-1) \cdot m^{n-1}=(n-1) \cdot \triangle N(m, n)$

The average degree $<k>$ can be defined as

$$
<k>=\frac{1}{N(m, n)} \sum_{j=1}^{N(m, n)} k_{j}
$$

where $k_{j}$ denotes the degree of node $j$.

The average degree $\langle k\rangle$ represents the average number of links per node, so in an equivalent way, it can be expressed as

$$
\begin{array}{r}
<k>=\frac{2 E(m, n)}{N(m, n)}=2\left[\left(1-\frac{1}{N(m . n)}\right)+(1-\right. \\
\left.\left.\frac{1+m}{N(m, n)}\right)+\cdots+\left(1-\frac{1+m+m^{n-2}}{N(m, n)}\right)\right]<2(n-1)
\end{array}
$$

Equations $(1,6)$ indicate that $N(m, n)$ increases exponentially with $m^{n-1}$ while $\langle k\rangle$ increases linearly with a number smaller than $2(n-1)$. Consequently, the average degree $\langle k\rangle$ remains relatively small compared to $N(m, n)$, even if the size of the network becomes extremely large. Figures 2 and 3 give a dramatic intuitive expression of this phenomenon.

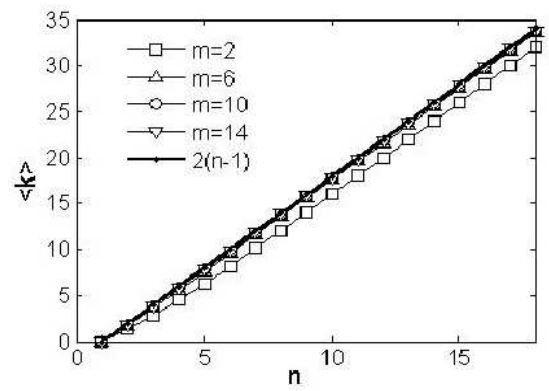

Fig. 3: Average degrees of the $m-n$ tree-like networks

In our model, it is obviously that the degrees of the same hierarchy nodes are identical. To calculate the node degree, we firstly introduce the following definitions.

Neighbor: node $i$ is a neighbor of node $j$ if there is an edge between node $i$ and $j$.

Upper-neighbor: neighbor node $i$ of node $j$ is called an upper-neighbor, if it belongs to an upper hierarchy than that of node $j$.

Lower-neighbor: neighbor node $i$ of node $j$ is called a lower-neighbor, if it belongs to a lower hierarchy than that of node $j$.

The question of calculating the degree $k_{j}$ of node $j$ now transforms into calculating the number of the upper-neighbors and lower-neighbors of node $j$. The number of the upper-neighbors $k_{j}^{u p p e r}$ and lower-neighbors $k_{j}^{\text {lower }}$ of node $j$ can be obtained by the following equations $(7,8)$ respectively.

$$
\begin{gathered}
k_{j}^{\text {upper }}=i-1 \\
k_{j}^{\text {lower }}=\frac{m\left(1-m^{n-i}\right)}{1-m}
\end{gathered}
$$

The degree $k_{j}$ of node $j$ now can be obtained using equation (9).

$$
k_{j}=k_{j}^{\text {upper }}+k_{j}^{\text {lower }}=i-1+\frac{m\left(1-m^{n-i}\right)}{1-m}
$$

\section{Static properties}

It has been proposed that many realistic networks share two common properties: small-world and scale-free properties. These two features of our proposed $m-n$ tree-like networks are verified in this section.

\subsection{The small-world effect}

A small-world network has a high clustering coefficient and a short average path length. Thanks to the deterministic nature of our model, we can solve it exactly. 


\subsubsection{Clustering coefficient}

Clustering, also known as transitivity is a typical property of acquaintance networks, where two individuals with a common friend are likely to know each other. Here we adopt the clustering coefficient definition of the graph clustering coefficient $\mathrm{C}$, a measure introduced by Watts and Strogatz [8], defined as equations $(10,11)$.

$$
\begin{gathered}
c_{j}=\frac{2 e_{j}}{k_{j}\left(k_{j}-1\right)} \\
C=<c_{j}>=\frac{1}{N(m, n)} \sum_{j=1}^{N(m, n)} c_{j}
\end{gathered}
$$

where $c_{j}$ denotes the clustering coefficient of the node $j$, $e_{j}$ denotes the actual links among the neighbors of node $j$, $k_{j}$ denotes the degree of node $j$, and hence $\left(k_{j}\left(k_{j}-1\right)\right) /(2)$ denotes the maximum possible links among the neighbors of node $j$. The clustering coefficient $C$ of the whole network is then given by the average of $\left\langle c_{j}>\right.$ over all nodes in equation (11).

To calculate the network clustering coefficient, we start from the node clustering coefficient. For a node $j$ in hierarchy $i$, the actual links $e_{j}$ among its neighbors can be calculated as the following three situations:

Firstly, calculate the links $e_{j 1}$ among its upper-neighbors. Using equation (7), we obtain

$$
e_{j 1}=\frac{(i-1)(i-2)}{2}
$$

Secondly, calculate the links $e_{j 2}$ among its lower-neighbors. In this case,

$$
e_{j 2}=m \cdot E(m, n-i)=m \sum_{i=0}^{n-i-1} i \cdot m^{i}
$$

Lastly, calculate the links $e_{j 3}$ among its upper-neighbors and lower-neighbors. For every node of the $m-n$ tree-like network, each of its upper-neighbors is directly linked to all its lower-neighbors. Using equations $(7,8)$, we obtain

$$
e_{j 3}=k_{j}^{\text {upper }} \cdot k_{j}^{\text {lower }}=\frac{m \cdot(i-1) \cdot\left(1-m^{n-i}\right)}{1-m}
$$

Using equations $(12,13,14)$, we obtain

$$
\begin{gathered}
e_{j}=e_{j 1}+e_{j 2}+e_{j 3}=\frac{(i-1)(i-2)}{2}+ \\
m \cdot \sum_{i=0}^{n-i-1} i \cdot m^{i}+\frac{m \cdot(i-1) \cdot\left(1-m^{n-i}\right)}{(1-m)}
\end{gathered}
$$

Substituting equations $(9,15)$ into equation(10), we can easily calculate the node clustering coefficient. As the node clustering coefficients of the same hierarchical are

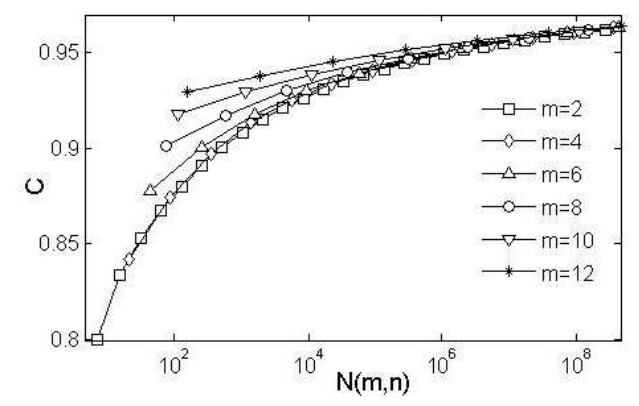

Fig. 4: Clustering coefficients of $m-n$ tree-like networks

equal, we can obtain the network clustering coefficient $C$ as follows.

$$
C=\frac{1}{N(m, n)} \sum_{j}^{N(m, n)} c_{j}=\frac{1}{N(m, n)} \sum_{i=1}^{n} C_{j}^{i} \cdot m^{i-1}
$$

where $C_{j}^{i}$ denotes the clustering coefficient of the nodes in hierarchy $i$.

Equation (16) accurately depicts the relationship between the clustering coefficient $C$ and the model parameters $m$ and $n$ in $m-n$ tree-like hierarchical network. Figure 4 shows the curves of the clustering coefficient $C$ increasing with the node size $N(m, n)$. It can be found that the clustering coefficient $C$ approaches an asymptotic value as $N(m, n)$ tends to infinity.

\subsubsection{Average path length}

In addition to a relatively high clustering coefficient, small-world networks have a short average path length. We can also exactly calculate the average path length $L$ for a given $m-n$ tree-like network. For a node $j$ of hierarchy $i$, its total path length $L_{j}$ with the other $N(m, n)-1$ nodes can be calculated as the following two parts:

The first part is the total path length $L_{j 1}$ with its neighbors.

$$
L_{j 1}=i-1+\frac{m\left(1-m^{n-i}\right)}{1-m}
$$

The second part is the total path length $L_{j 2}$ with the nodes besides its neighbors. The minimum links between these nodes and node $j$ is 2 , because they can reach node $j$ through the main root node.

$$
L_{j 2}=2 \times\left(\frac{\left(1-m^{n}\right)-m\left(1-m^{n-i}\right)}{1-m}-i\right)
$$

Therefore, the total path length $L_{j}$ between node $j$ and the other nodes can be stated as follows

$$
L_{j}=L_{j 1}+L_{j 2}=\frac{2\left(1-m^{n}\right)-m\left(1-m^{n-i}\right)}{(1-m)}-i-1
$$




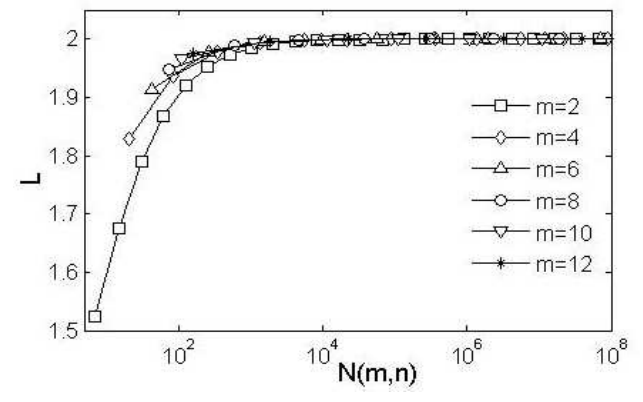

Fig. 5: Average path length of $m-n$ tree-like networks

Note that the node number of hierarchy $i$ is $m^{n-i}$, and the number of node pairs is $C_{N(m, n)}^{2}$. Hence, we can calculate the average path as follows

$$
L=\frac{1}{2} \cdot \frac{1}{C_{N(m, n)}^{2}} \cdot \sum_{i=1}^{n} L_{j} \cdot m^{i-1}
$$

The curves of the average path length $L$ increasing with the network size $N(m, n)$ are depicted in Figure 5. We can find that the average path length $L$ approaches an asymptotic value 2 as $N(m, n)$ tends to infinity.

\subsection{The scale-free property}

The scale-free network has a highly inhomogeneous degree distribution, i.e., it simultaneously has a few nodes connecting many other nodes and a large number of poorly connected ones [9]. In our $m-n$ tree-like network, the probability of a random selected node $j$ belonging to hierarchy $i$ can be calculated as follows.

$$
P\left(k^{i}\right)=\frac{m^{i-1}}{N(m, n)}=\frac{m^{i-1} \cdot(1-m)}{1-m^{n}}
$$

Substituting equation (9) into (21), we can obtain the discrete degree distribution as the following expressions.

$$
\begin{gathered}
P\left(i-1+\frac{m\left(1-m^{n-i}\right)}{(1-m)}\right)=\frac{m^{i-1} \cdot(1-m)}{1-m^{n}} \\
P(k)=\frac{m^{i} \cdot\left(1-m^{n-i}\right)}{k\left(1-m^{n}\right)}-i+1
\end{gathered}
$$

The degree distributions of two illustrative $m-n$ treelike network examples are shown in Figure 6 and Figure 7. It can be seen that the $m-n$ tree-like network has a fattailed degree distribution as many other acquainted scalefree networks.

\section{Tree-like cascading failures model}

To investigate the dynamic of cascading failures in $m-n-s$ tree-like networks, we first propose a weighted cascading failures model in this section.

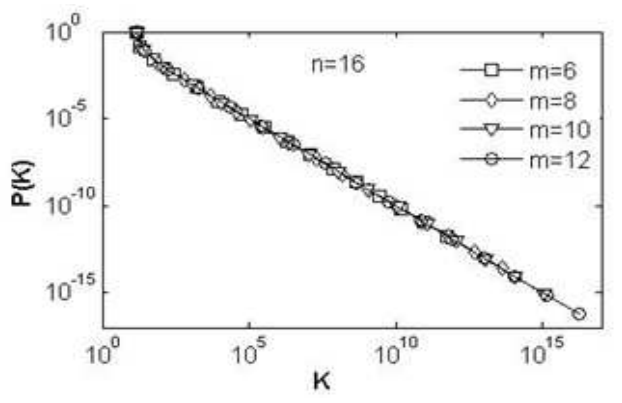

Fig. 6: Degree distribution of $m$-16 tree-like networks with $m=$ $6,8,10,12$

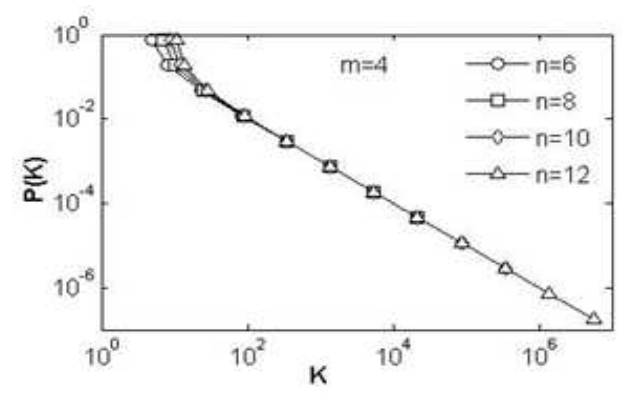

Fig. 7: Degree distribution of 4- $n$ tree-like networks with $n=$ $6,8,10,12$

\subsection{Initial load definition}

The node load has been studied in the past as a measure of the node centrality and influence. It is often defined to be the function of the node degree. However, in our $m-n-s$ tree-like networks, the node hierarchy also has important effect on node load. Therefore, we define the initial node load $L_{j}$ as the coupling function of its degree $d_{j}$ and hierarchy $h_{j}$ as follows:

$$
L_{j}=d_{j} \cdot\left(n-h_{j}+1\right)^{\alpha}
$$

where $h_{j}$ denotes the hierarchy of node $j, n$ is the hierarchies of the whole network, $\alpha \geq 0$ denotes the hierarchy weight, and it is a variable parameter. It is obvious that the bigger $\alpha$ is, the bigger weight the hierarchy is. Hereafter, we call $\alpha$ as hierarchy weight parameter.

\subsection{Node capacity}

Following previous models, each node has a capacity threshold, which means the maximum load that it can handle. Since the node capacity in man-made networks is severely limited by cost, it is natural to assume that the capacity $C_{j}$ of node $j$ is proportional to its initial load

$$
C_{j}=(1+\beta) \cdot L_{j}
$$


where $L_{j}$ denotes the initial load of node $j, \beta>0$ is a tunable parameter. Hereafter, we call $\beta$ as node capacity parameter.

\subsection{Load redistribution}

After one node failures initially, its previous load or function must be distributed to its reactive neighbor nodes to maintain the network's function. If these neighbor nodes cannot afford the additional load, they will become new failure nodes, and cause the next load redistribution step. Now, we need to answer the question of how to distribute the previous load of failure nodes to their reactive neighbor nodes. In our model, we adopt the simple rule of "able people should do more work". That is, the previous load of the failure nodes is assigned to their active neighbor nodes according to the neighbors' capacities.

The load redistribution rule can be stated as follows

$$
\Pi_{j}=\sum_{i \in \Phi} L_{i} \times\left(\frac{C_{j}}{\sum_{i \in \Gamma} C_{j}}\right)
$$

where $\Pi_{j}$ denotes the additional load assigned to the reactive neighbor node $j, \Phi$ denotes the set of all failure nodes, $L_{i}$ denotes the previous load of failure node $i, \Gamma$ denotes the set of all reactive neighbor nodes, $C_{j}$ denotes the capacity of reactive neighbor node $j$.

\subsection{Robustness measure}

It can be seen that the failure of one node may cause the whole network collapse due to the cascading failure. On the other hand, it may have little influence on the whole network. To distinguish this phenomenon, we introduce the conception of node cascading failure size.

Node cascading failure size $\left(C F_{i}\right)$ : the network cascading failure size caused by the initial failure of node $i$, which can be calculated as follows.

$$
C F_{i}=\frac{\left(F_{i}-1\right)}{N-1}
$$

where $F_{i}$ denotes the number of all failure nodes, $N$ denotes the node number of the whole network.

Based on the above conception of $C F_{i}$, we furthermore introduce the conception of network cascading failure size.

Network cascading failure size $(C F)$ : the average cascading failure size caused by the initial failure of each node, which can be calculated as follows.

$$
C F=\sum_{i \in N} \frac{C F_{i}}{N(N-1)}
$$

where $C F_{i}$ denotes the node cascading failure size of node $i, N$ denotes the set of network nodes.
It is obvious that the network cascading failure size $C F(0 \leq C F \leq 1)$ reflects the average remaining function of the whole network due to the cascading failures. $C F=0$ denotes the normal function of the network, and $C F=1$ denotes the absolutely collapse.

Noteworthy is the fact that, as the $\beta$ increases, a phase transition could occur at the critical threshold $\beta_{c}$. When $\beta>\beta_{c}$, no cascading failure occurs and the network maintains its normal function, i.e., $C F=0$; and when $\beta<\beta_{c}$, cascading failure emerges, i.e., $C F>0$. In other words, the critical threshold $\beta_{c}$ is the least protection strength to avoid cascading failure. Here, we use $\beta_{c}$ to evaluate the network robustness against cascading failure. Apparently, the smaller the $\beta_{c}$, the more robust the network.

\section{Robustness against cascading failures}

Up to now, it has been shown again and again that the topological structure of complex network has great impacts on its dynamics $[10,11]$. Here, we investigate the impacts of the tree-like hierarchical structure on dynamics of cascading failure. We first calculate the robustness measure by theoretical analysis, and furthermore give an illustrative simulation.

\subsection{Theoretical analysis}

To avoid the emergence of cascading failures, the following condition should be satisfied:

$$
L_{j}+\triangle L_{j}<C_{j}
$$

where $L_{j}$ denotes the initial load of the reactive neighbor node $j, \triangle L_{j}$ denotes the additional load assigned to node $j$ during the cascading failure procedure, $C_{j}$ denotes the capacity of node $j$.

Substituting equations $(24,25,26)$ into equation (29), we obtain

$$
1+\frac{d_{i} \cdot\left(n-h_{i}+1\right)^{\alpha}}{\sum_{j \in \Gamma} d_{j} \cdot\left(n-h_{j}+1\right)^{\alpha}}<\beta
$$

where $d_{i}$ and $d_{j}$ respectively denotes the node degree of the failure node $i$ and reactive neighbor node $j, h_{i}$ and $h_{j}$ respectively denotes the hierarchy of failure node $i$ and reactive neighbor node $j, n$ is the hierarchies of the whole network, $\Gamma$ denotes the set of all reactive neighbor nodes.

Now, we can calculate the node critical threshold $\beta_{c}^{i}$ as follows

$$
\beta_{c}^{i}=\max \left(1+\frac{d_{i} \cdot\left(n-h_{i}+1\right)^{\alpha}}{\sum_{j \in \Gamma} d_{j} \cdot\left(n-h_{j}+1\right)^{\alpha}}\right)
$$

Finally, we obtain the network critical threshold

$$
\beta_{c}=\max _{j \in N}\left\{\beta_{c}^{j}\right\}
$$




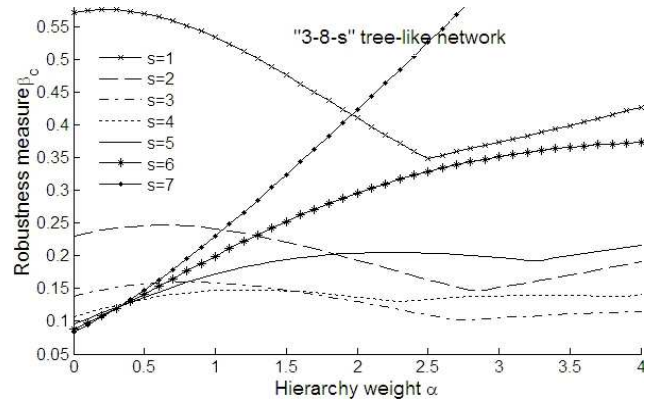

Fig. 8: The impact of topology structure on network robustness

Obviously, the nodes in the same hierarchy have the same degree, the same hierarchy and hence the same critical threshold. That facilitates the calculation of the robustness measure by using the equations $(31,32)$.

\subsection{Illustrative simulation}

In last section, we discussed the theoretical calculation of the network robustness $\beta_{c}$. Now we can investigate the relationship between topology structure and the dynamic of cascading failures. In our weighted tree-like cascading failures model, two parameters impact the network topology: the skipping-ability $s$, which determines the nodes' degree, and the hierarchy weight $\alpha$, which determines the network characteristic of weighted hierarchy. To investigate their impacts on the network robustness $\beta_{c}$, a set of illustrative simulations based on "3-8-s" tree-like networks are proposed in this section. The formulas that will be derived in the following section can easily be adapted to deal with any other " $m-n-s$ " tree-like networks.

The simulation results of the " $3-8-s$ " tree-like networks are depicted in Figure 8. As can be seen in Fig. 8 , the ordinate axis $\beta_{c}$ denotes the least protection strength to avoid cascading failure, and the smaller the $\beta_{c}$, the more robust the network. The abscissa axis $\alpha$ denotes the hierarchy weight, and the bigger the $\alpha$, the more hierarchy weighted the network. The seven curves respectively denote the seven "3-8-s" topology structures with the skipping-ability $s=1,2, \cdots, 7$.

We first investigate the relationship between skipping-ability and network robustness against cascading failures. Comparing the seven curves in Figure 8, we can find that, the skipping-ability has important impacts on the network robustness. The smallest skipping-ability, i.e., $s=1$, and the relatively bigger skipping-ability, for example, $s=6$ and $s=7$, correspond to the relatively less robust networks. Oppositely, the compromise skipping-ability values $s=3$ and $s=4$ correspond to the most robust networks. To analyze the reason, we can find that the node capacities of the smallest skipping-ability networks are too small to protect against the cascading failures; on the other hand, the node initial loads of the biggest skipping-ability networks are too big, it is easy to occur cascading failures when one node initially failures.

Next, we analyze the impacts of the hierarchy weight on the network robustness against cascading failures. As can be seen in Figure 8, two conclusions are obvious. Firstly, for the relatively bigger skipping-ability networks, for example, $s=6$ and $s=7$, the bigger the hierarchy weight, the less robust the network. This is obvious, because the more weighted the network, the more easily the cascading failures occur caused by the failure of the top hierarchy nodes. Furthermore, we can obvious another interesting and counterintuitive result. For the relatively smaller skipping-ability networks, for example, $s=1$ and $s=2$, the relationship curves present the ' $\mathrm{V}$ ' shapes. In other words, there is a best hierarchy weight value causing the network mostly robust. These simulation conclusions may help us better understand the cascading phenomena in the real tree-like hierarchical networks.

\section{Conclusions}

To investigate the properties of tree-like hierarchical structure, we first propose a deterministic tree-like hierarchical network model. Secondly, the small-world and scale-free properties of our proposed tree-like network are discussed. The simulation and theoretical analysis prove that our proposed tree-like hierarchical networks conformably possess the small-world and scale-free properties well.

Furthermore, we investigate the dynamic of cascading failures on tree-like hierarchical networks. A weighted tree-like cascading failure model is first proposed, and the relationship between the tree-like structure function and the networks robustness is investigated by a set of illustrative simulations. We find some interesting and counterintuitive simulation conclusions, which may be helpful for the further studying of the tree-like networks.

\section{Acknowledgement}

This work is partially supported by the National Natural Science Foundation of China (Grant No. 71101149, 71201169, 71001105, 71031007) and the Natural Science Foundation of Hunan Province, China (Grant No. 11JJ4065).

\section{References}

[1] A. V. Aho and J. D. Ullman, Principles of Compiler Design (Addison-Wesley series in computer science and information processing), Addison-Wesley Longman Publishing Co., (1977). 
[2] T. H. Cormen, C. E. Leiserson, R. L. Rivest and C. Stein, Introduction to algorithms, MIT press, (2001).

[3] E. Ravasz and A. L Barabsi, Physical Review E, 67, 026112 (2003).

[4] L. F Costa, Physical review letters, 93, 098702 (2004).

[5] A. L Barabsi and T. Vicsek, Physica A: Statistical Mechanics and its Applications, 299, 559-564 (2001).

[6] Z. X Wu, G. Peng, W. X Wang, S. Chan, and E. M Wong, Journal of Statistical Mechanics: Theory and Experiment, 5, P05013 (2008).

[7] M. Chen, B. Yu, P. Xu and J. Chen, Physica A: Statistical Mechanics and its Applications, 385, 707-717 (2007).

[8] D. G Watts and S. H Strogatz, nature, 393, 440-442 (1998).

[9] S. Boccaletti, V. Latora, Y. Moreno, M. Chavez and D. U Hwang, Physics reports, 424, 175-308 (2006).

[10] J. Wang, L. Rong, L. Zhang and Z. Zhang , Physica A: Statistical Mechanics and its Applications, 387, 6671-6678 (2008).

[11] W. Wang and G. Chen, Physical Review E, 77, 026101 (2008).

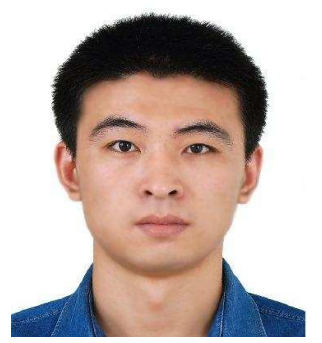

Yingxin Zhang received the MS degree in Military Operation Research science from National University of Defense Technology in 2009. He is currently a Ph.D. candidate in Department of Information System and Management, National University of Defense Technology. His research interests are in the areas of network architecture, complexity of war system, and battle modeling and simulation.

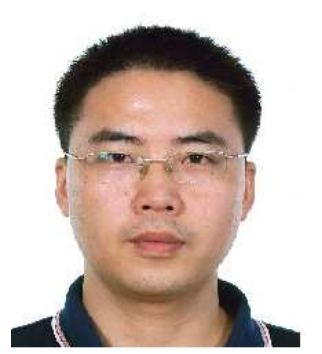

Chao Chen was born in 1977. He received his M.S. and Ph.D. degrees from National University of Defense Technology in 2003 and 2007. He is now a lecturer in National University of Defense Technology. His research interests include complexity of war systems, battle modeling and simulation, and automatization in command and control system.

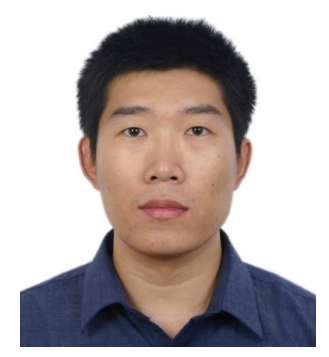

Jianmai Shi was born in 1980. He received his M.S. and Ph.D. degrees from National University of Defense Technology in 2005 and 2011. He is now a lecturer in National University of Defense Technology. His research interests include complexity of war systems, mission planning, and supply chain management. 